showed an increased excretion of 2-ketoglutaric acid and 3-hydroxyisovaleric acid. Serum biotinidase activity was $0.15 \mathrm{nmol} \mathrm{min}^{-} \mathrm{ml}^{2}$ (normal range 5.2). Father's biotinidase activity was 0.31 ( $8 \%$ of normal) and the mother's 0.42 ( $10 \%$ of normal). An electroencephalogram showed frequent independent spikes of variable amplitude prominent in the left posterior temporal region and numerous EEG seizures. Within 36 hours of starting biotin therapy $5 \mathrm{mg} \mathrm{BD}$ there was dramatic clinical improvement; the infant became responsive to surrounding stimuli, seizures were controlled and antiepileptic treatment was reduced to only phenobarbital $15 \mathrm{mg} \mathrm{BD}$. After ten days of treatment the urinary examination was normal and the EEG showed a well organized background activity and no paroxysmal abnormalities. At two years four months the neurological exam was normal, the CT scan and EEG normal, and the dose of biotin was at $7.5 \mathrm{mg}$ a day. The authors suggest a therapeutic trial of biotin in all drug resistant infantile seizures. (Colamaria V et al. Biotin-responsive infantile encephalopathy: EEG-polygraphic study of a case. Epilepsia October 1989; $\underline{30: 573-578) .}$

COMENT. Two forms of biotin responsive encephalopathy are reported. 1) neonatal holocarboxalase synthetase deficiency (HCSD) and 2) late onset infantile or juvenile biotinidase deficiency (BD). HCSD patients have vamiting, lethargy, and hypotonia associated with metabolic ketoacidosis, hyperamonemia, and organic acidemia. BD infants present with seizures, ataxia, skin rash and alopecia. Seizures are reported in two of five HCSD cases and in 15 of $28 \mathrm{BD}$ cases. The authors stress that the epileptic symptomatology may be the first clinical feature in BD cases. Myoclonias, auditory myoclonus, and repetitive startles documented in the present case were thought to be nonepileptic in nature.

\title{
LANGUAGE DISORDERS AND EPILEPSY
}

\section{OROMDIOR DYSPRAXIA IN BENIGN OHILDHOOD EPILEPSY}

A six year old right handed boy with prolonged intermittent drooling, oromotor dyspraxia, and benign childhood epilepsy with centrotemporal spikes is reported from the Departments of Pediatrics and Neurology, Centre Hospitalier, Universitaire Vaudois, Lausanne, Switzerland. Seizures began on the third day of 1 ife and were controlled wi th phenobarbital. Febrile seizures began at eight months and recurred 12 times up to six years of age. At first the seizures were generalized but after four years of age they were partial motor, involving the face and sametimes the right arm. The drooling probably resulted from hypersalivation and oramotor dyspraxia. The fluctuant course of the symptams and correlation with intensity of the paroxysmal discharges on EEG were consistent with an epileptic dysfunction located in the lower rolandic fissure. No lesion was demonstrated by MRI. (Roulet E, Deonna T, Despland PA. Prolonged intermittent drooling and oromotor dyspraxia in benign childhood epilepsy with centrotemporal spikes. Epilepsia October 1989, 30:564-568). 
COMMENT. This case provides evidence that a continuous epileptic dys function can occur in benign childhood epilepsy with cent rotemporal spikes. The case resembles the acquired aphasia epilepsy syndrome of Landau-Kleffner.

\section{LANDAU-KLEFFNER SINDRCME AND NEUROCYSTICERCOSIS}

A seven year old right-handed boy with a one year history of language disorder associated with clinical seizures and paroxysmal EFG is reported from the Division de Neurologia, Instituto Nacional de Neurologia Y Neurocirugia, Mexico, D.F. He had normal speech and comprehension up to age six when he developed several brief episodes of loss of awareness and unresponsiveness associated with autamatic movements of the hands, recurring more than 20 times a day. Two months later he did not respond to calls and did not comprehend stories read to him. His speech became telegraphic. CT and MRI revealed a small cysticercus deep in the left Sylvian fissure. An EFG showed sharp and slow wave complexes over the left centrotemporal regions with spread to the right side. Treatment with the anticysticercal drug Albendazole resulted in reduction in size of the cyst, valproate controlled the seizures, and the language disorder improved. Follow-up at six months showed no recurrence. (Otero $\mathrm{E}$ et al. Acquired epileptic aphasia (the Landau-Kleffner syndrome) due to neurocysticercosis. Epilepsia October $1989 ; 30: 569-572$ ).

COMMENT. Deonna $\mathrm{T}$ and associates from the Pediatric Department, Lausanne, Switzerland, report an adult follow-up study of seven patients with acquired aphasia-epilepsy syndrome beginning in childhood (Neuropediatrics, August 1989, 20:132-138). One man had recovered completely, one had normal oral language but was severely dyslexic, one recovered normal comprehension but had severe expressive language problems, four had absent language comprehension and lack of expressive speech. Only one had learned and is using sign language with some efficiency, and none had developed functional written language. Attempts to offer a substitutive language to children with acquired aphasia-epilepsy syndrome were not very successful. Isolated reports of improvement with anticonvulsant drug treatment justified further trials. Ansink BJJ et al fram the Sint Lucas Ziekenhuis, Amsterdam, the Netherlands, report a child who developed seizures with fever at 20 months of age. The fever was caused by pneumonia. Four months later she had fever and status epilepticus which were followed by abnormal behavior and aphasia. The aphasia was complicated by visual agnosia and spatial disturbances. The EEG showed mult ifocal epileptic activity. Treatment with valproic acid controlled the seizures and language functions recovered slowly. At a recent meeting of the American Epilepsy Society there were two papers on the Landau-Kleffner syndrome: 1) Marescaux C et al from Strasbourg, France and Liege, Belgium described two patients wi th a syndrome of continuous spike wave discharges during sleep associated with aphasia; and 2) Morrell F et al from Rush Medical College, Chicago, reported two patients with the syndrome who were treated successfully by multiple transection. 